\title{
ТЕХНОЛОГИИ АТМОСФЕРНОЙ ЭЛЕКТРОДИНАМИКИ НА ОСНОВЕ ИОНИЗИРОВАННЫХ ДИСПЕРСНЫХ СРЕД
}

\author{
И.Н. Абросимов, Н. И. Абросимов, Л. М. Макальский, Т. Ю. Фокин \\ ИГОРЬ НИКОЛАЕВИЧ АБРОСИМОВ - к.т.н., дочент Российского технологического университета \\ МИРЭA. E-mail:abrosimov@mirea.ru. \\ НИКОЛАЙ ИГОРЕВИЧ АБРОСИМОВ - зав. сектором ФГБУ Научно-исследовательского иентра \\ «Планета».
}

ЛЕОНИД МИХАЙЛОВИЧ МАКАЛЬСКИЙ - к.т.н., доџент ФГБОУ ВО «МЭИ - Национальный исследовательский университет».

ТИМОФЕЙ ЮРЬЕВИЧ ФОКИН - магистрант Российского технологического университета МИРЭА.

119454, Москва, Россия, Проспект Вернадского, д. 78. Российский технологический университет МИРЭА.

123242, Москва, Россия, Большой Предтеченский пер., д. 7. ФГБУ Научно-исследовательский иентр «Планета».

111250, Москва, Россия, Красноказарменная ул., д. 14. ФГБОУ ВО «МЭИ - Национальный исследовательский университет».

Рассмотрены параметры ионизированных дисперсных микрочастии, образованных гидридными соединениями, одним из которых является вода. Свойства соединений водорода и химических элементов 5, 6, 7 групп 2 периода отличаются по температурам фазовых переходов от других соединений, не смотря на подобие s и р электронных орбит. Рассмотрены условия ионизации микрочастии воды в коаксиальном резонаторе и исследованы траектории их движения в атмосфере. Поведение микрочастии аналогичны свойствам волн де Бройля, что позволяет использовать их возможности в информачионных и других технологиях.

Ключевые слова: ионизация, дисперсные микрочастицы, резонатор, корпускулярно-волновые, электрический разряд, температурный переход, конденсированная среда.

\section{ATMOSPHERIC ELECTRODYNAMICS TECHNOLOGIES BASED ON IONIZED DISPERSED MEDIA}

\author{
I. N. Abrosimov ${ }^{1}$, N. I. Abrosimov², L. M. Makalsky³, T. Y. Fokin ${ }^{1}$ \\ ${ }^{178}$ Prospekt Vernadskogo, Moscow, 119454, Russia. MIREA - Russian Technological University. \\ 27 Bolshoy Predtechenskiy per., Moscow, 123242, Russia. Scientific-Research center «Planeta». \\ ${ }^{3} 14$ Krasnokazarmennaya str., Moscow, 111250, Russia. Moscow power engineering Institute national research \\ University
}


The parameters of ionized dispersed microparticles formed by hydride compounds, one of which is water, are considered. The properties of hydrogen compounds and chemical elements of groups 5, 6, and 7 of period 2 differ in the temperatures of phase transitions from other compounds, despite the similarity of s and p electron orbits. The conditions of ionization of water microparticles in a coaxial resonator are considered and the trajectories of their movement in the atmosphere are studied. The behavior of microparticles is similar to the properties of de Broglie waves, which makes it possible to use their capabilities in information and other technologies.

Keywords: ionization, dispersed microparticles, resonator, particle-wave, electric discharge, temperature transition, condensed medium.

Дисперсные частицы малых размеров могут образовываться из жидких и твердых конденсированных сред. Эти частицы можно считать одним из физических состояний конденсированных сред, которые сохраняют или принимают твердую, жидкую или парообразную агрегатную форму. В отличие от однородной устойчивой массы вещества дисперсные частицы являются неоднородными и нестабильными образованиями. Причиной появления дисперсных частиц могут быть природные и техногенные процессы, которые могут носить позитивный или негативный характер.

Существуют различные физические методы создания дисперсных частиц. Такими методами могут быть механические, ультразвуковые, термические, пневматические, электрокапиллярные, трибоэлектрические, оптоакустические и другие. Механические устройства имеют максимальную производительность, термические дают возможность получить микрочастицы молекулярных размеров, в ультразвуковых методах размеры частиц однозначно связаны с частотой акустических нелинейный колебаний, работа других устройств основана на других физических принципах действия, которые характеризуются своими достоинствами и ограничениями.

В зависимости от используемого метода и его технической модификации могут быть получены дисперсные частицы микронных и субмикронных размеров. Дисперсные частицы, образованные из конденсированных сред, получили название аэрозолей [1]. Аэрозоли нашли широкое применение во многих областях науки и техники.

В данной работе внимание уделено дисперсным частицам (аэрозолям) на основе гидридов, представляющих собой соединения водорода с химическими элементами 6 группы. Основное рас- пространение имеет гидрид кислорода $\mathrm{H}_{2} \mathrm{O}$, являющийся соединением водорода и кислорода. Другие гидридные соединения, образованные водородом и остальными элементами 6 группы, используются значительно меньше. Интерес к другим гидридам обусловлен сходством электронных структур. К ним относятся соединения водорода с полонием (Рo), теллуром (Te), селеном $(\mathrm{Se})$, серой $(\mathrm{S})$ и с упомянутым выше кислородом (О). Эти элементы имеют подобные оболочки с электронной структурой s2p4, на которых находятся по 6 электронов. Внешние оболочки элементов 6 группы приведены в таблице 1 .

Согласно закономерностям периодической таблицы свойства элементов и их соединений, в основном согласованы с атомным весом и расположением в периодах и группах. Значения атомных весов гидридов отражено в неравенстве $\mathrm{H}_{2} \mathrm{Po}>\mathrm{H}_{2} \mathrm{Te}>\mathrm{H}_{2} \mathrm{Se}>\mathrm{H}_{2} \mathrm{~S}>\mathrm{H}_{2} \mathrm{O}$, которое характеризуется линейной закономерностью термодинамических характеристик за исключением воды $\mathrm{H}_{2} \mathrm{O}$. Остальные гидриды этой группы имеют низкие температуры образования жидкой и твердой фаз в соответствии с атомным весом и номером элемента. Таким образом, отличительные свойства воды $\mathrm{H}_{2} \mathrm{O}$ от других гидридов дают основания считать её по физическим свойствам уникальным аномальным соединением. Связь термодинамических характеристик гидридов с атомным весом иллюстрирует рисунок 1 .

Среди элементов гидридных соединений кислород обладает наибольшей реакционной способностью. В этой подгруппе $\mathrm{O}-\mathrm{S}-\mathrm{Se}-\mathrm{Te}-\mathrm{Po}$ с увеличением атомного веса происходит изменение физических свойств с диэлектрических на проводящие. Вода является наиболее важным соединением, структура которой описана ниже.

Таблица 1

Внешние электронные орбиты элементов 6 группы, образующие гидридные соединения с водородом

\begin{tabular}{cccccc}
\hline Элементы & ${ }^{8} \mathbf{O}_{16.0}$ & ${ }^{16} \mathbf{S}_{32.06}$ & ${ }^{34} \mathbf{S e}_{78.69}$ & ${ }^{52} \mathbf{T e}_{127.6}$ & ${ }^{84} \mathbf{P o}_{210}$ \\
\hline Энергетические уровни & $\mathrm{L}$ & $\mathrm{M}$ & $\mathrm{N}$ & $\mathrm{O}$ & $\mathrm{P}$ \\
\hline
\end{tabular}




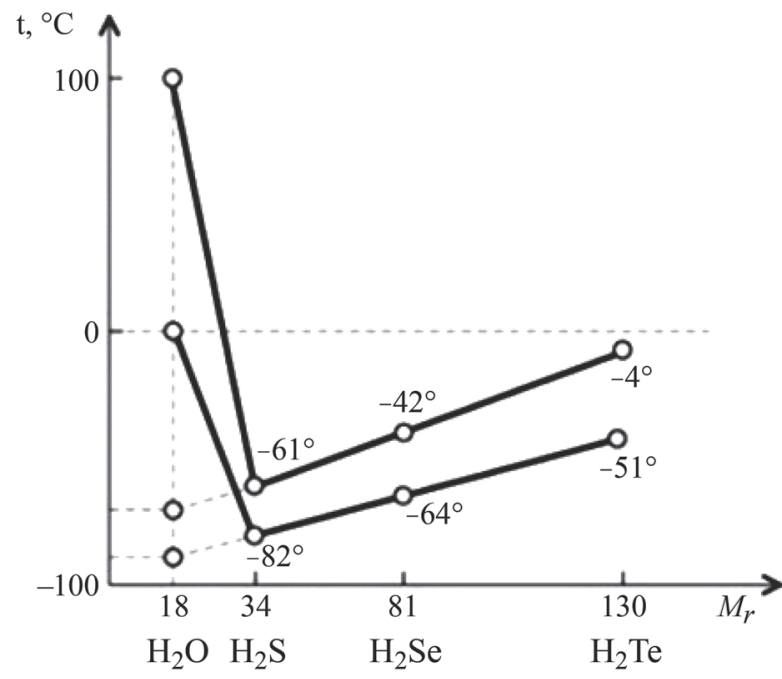

Рис. 1. Значения температур фазовых переходов гидридов для твердого, жидкого и газообразного (парообразного) состояний

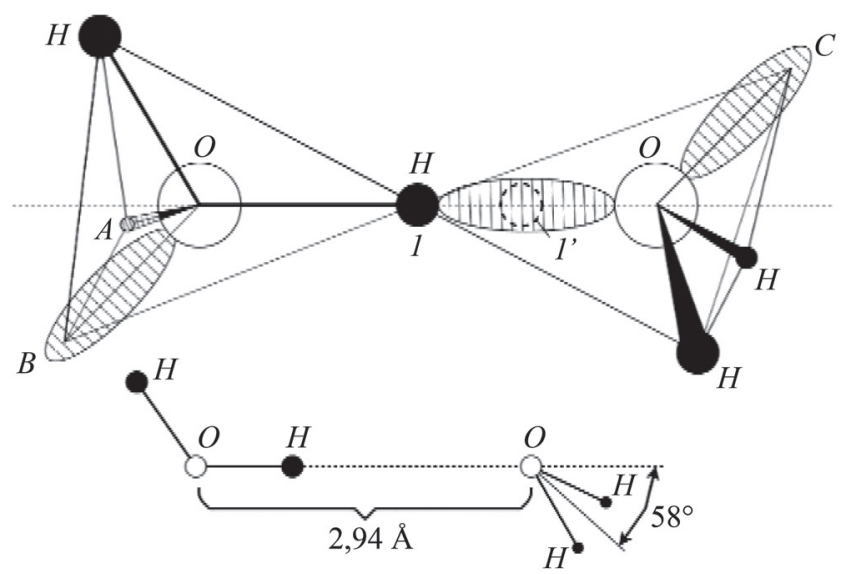

Рис. 2. Структура димера воды

Молекула воды является объединением водорода и кислорода. Стабильным соединением водорода и кислорода является димер воды $\left(\mathrm{H}_{2} \mathrm{O}\right)_{2}$, структура которого приведена на рисунке 2 [2].

Две молекулы воды объединены водородной связью с помощью атома водорода, который осциллирует между атомами кислорода обеих молекул, занимая попеременно позиции 1 и 1'. Считают, что димер может образовывать до 6 водородных связей с другими молекулами воды.

Размеры атомов водорода примерно равны $10^{-10} \mathrm{M}$, а кислорода $1,36 \cdot 10^{-10} \mathrm{M}$. Потенциалы ионизации водорода и кислорода имеют близкую величину, равную 13,6 эВ. Закономерности уменьшения размеров атомов в периоде и увеличения их размеров в группе периодической таблицы обусловлены четырьмя квантовыми числами в соответ- ствии с принципом запрета Паули, справедливым для фермионов [3].

Значительное внимание к воде уделяется в результате её важности в природе и человеческой жизнедеятельности. Задачей нашей работы является получение «холодной» плазмы на основе воды и её растворов. Получение заряженных водных микрочастиц возможно при преобразовании жидкой среды в аэрозоли. Малый размер аэрозолей позволяет создать на них электрические заряды. Наличие одноимённых электрических зарядов на аэрозолях препятствует объединению частиц и открывает возможность управлять скоростью и направлением их движения с помощью электрических и магнитных полей. Заряд воздушных и водных частиц может осуществляться с помощью электрической ионизации. Одним из методов ионизации является применение режима коронного разряда. Значения напряженности электрического поля, необходимого для осуществления перехода самостоятельного разряда в коронный, приведены ниже [4].

При положительной полярности электрода значение напряженности Е равно:

$$
E^{(+)}=33,7 \delta\left(1+\frac{0,242}{\sqrt{\delta R}}\right)
$$

При отрицательной полярности электрода:

$$
E^{(-)}=31,02 \delta\left(1+\frac{0,308}{\sqrt{\delta R}}\right),
$$

где $\delta=0,392 p / T-$ приведённая плотность упругой среды, $p$ - давление, $T$ - температура, $R$ - радиус электрода.

Причём напряженность поля $E$ может быть постоянной величиной или изменяться во времени.

Зарядное устройство состоит из двух электродов, один из которых стремятся выполнить с минимальными размерами. Конструкцию выше указанного устройства можно выполнить в виде цилиндрического коаксиального конденсатора, волновое сопротивление $\mathrm{Z}$ которого зависит от значений погонной индуктивности $\mathrm{L}$ и ёмкости $C$ :

$$
\begin{aligned}
& \mathrm{L}=\frac{\mu}{2 \pi} \ln \frac{R_{2}}{R_{1}}\left(\Gamma_{\mathrm{H}}\right), \\
& C=\frac{2 \pi \varepsilon}{\ln \frac{R_{2}}{R_{1}}}(\Phi),
\end{aligned}
$$

где $\mathrm{L}$ и $C$ зависят от $R_{1}, R_{2}$ внутреннего и наружного радиусов конденсатора. Элементы зарядного устройства приведены на рисунке 3. 


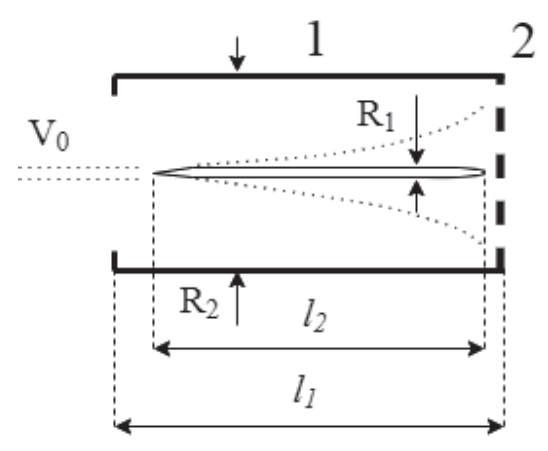

Рис. 3. Схема зарядного устройства микрочастиц

Зарядное устройство содержит коаксиальный конденсатор 1 , состоящий из центрального и наружного электрода. Иногда целесообразно применять управляющий электрод 2 электрически изолированный от электродов резонатора и прозрачный для микрочастиц. Между электродами создается электростатическое поле с напряженностью Е. Это поле необходимо для возникновения электрических зарядов на пролетающих в конденсаторе микрочастицах. При воздействии электрического поля происходит изменение траектории частиц, которая обусловлена уравнением движения:

$$
\mathrm{m} \frac{\mathrm{d}^{2} \mathrm{r}}{\mathrm{d}^{2} \mathrm{t}}=q E
$$

где $\mathrm{m} \frac{\mathrm{d}^{2} \mathrm{r}}{\mathrm{d}^{2} \mathrm{t}}$ - сила Ньютона, а $q E$ - сила Кулона.

В результате воздействия электрического поля на микрочастицы, в них возникает радиальная составляющая скорости, которая приводит к угловому расширению пучка. Угловое расширение зависит от соотношения исходной продольной и радиальной составляющих скоростей микрочастиц. Частичное угловое расширение пучка микрочастиц можно скомпенсировать применением соленоида с продольным магнитным полем [5]. В этом случае имеет место вращательная циркуляция микрочастиц вокруг оси с сохранением продольного перемещения. Радиальное отклонение пучка $\Delta \mathrm{r}$ зависит от значения напряженности электрического поля, параметров заряженных микрочастиц и конструкции коаксиального конденсатора:

$$
\Delta \mathrm{r}=\frac{q}{m v^{2}} \int_{0}^{l_{2}} l_{2} E d z
$$

Движение заряженных микрочастиц приводит к возникновению конвекционного тока I, величина которого будет равна произведению плотности зарядов $\rho$ на продольную составляющую скорости $v$ :

$$
I=\rho v=\mathrm{N} q v
$$

где $\mathrm{N}$ - количество заряженных микрочастиц.

Появление заряженных частиц в коаксиальном конденсаторе изменяет его электродинамические параметры. При отсутствии электрического заряда на частицах волновое сопротивление коаксиального конденсатора совпадает с волновым сопротивлением свободного пространства $Z_{0}$, которое равно отношению магнитной $\mu_{0}$ и диэлектрической $\varepsilon_{0}$ проницаемостей.

$$
Z_{0}=\sqrt{\frac{\mu_{0}}{\varepsilon_{0}}}
$$

Его значение для свободного пространства равно 376,7 Ом. Возникновение зарядов на микрочастицах приводит к изменению диэлектрической $\varepsilon$ и магнитной $\mu$ проницаемостей. В результате в коаксиальном резонаторе воздушное заполнение заменяется плазменным. Появление заряженных частиц с концентрацией $\mathrm{N}$ в объёме конденсатора меняет его частоту и волновое сопротивление, для расчёта которого можно использовать следующую формулу:

$$
\mathrm{Z}_{0}^{\prime}=\frac{1}{2 \pi} \sqrt{\frac{\mu}{\varepsilon}} \ln \frac{R_{2}}{R_{1}}
$$

Возможными методами увеличения концентрации заряженных частиц являются модуляция этих частиц по скорости или накопление объёмного заряда в локальной области. На рисунке 4 приведены траектории электрических разрядов с наличием объёмного заряда (рис. 4а) и без него (рис. 4б).

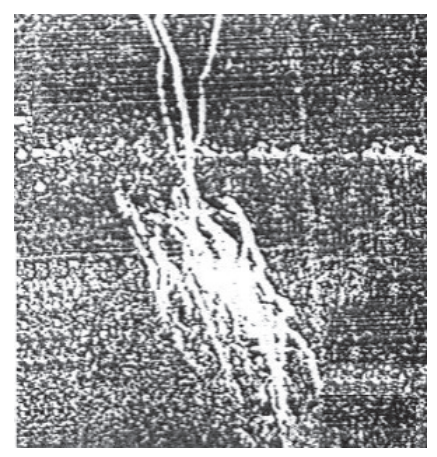

a

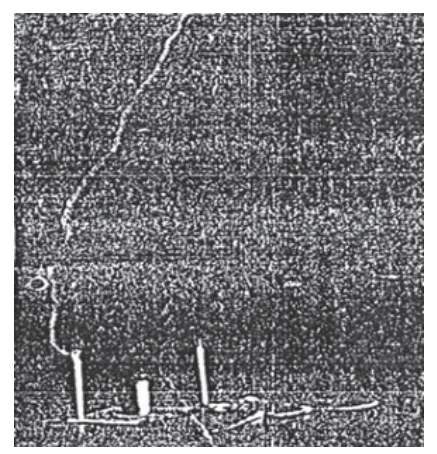

б
Рис. 4. Фотографии электрических разрядов потоков заряженных микрочастиц 
Сравнение изображений электрических разрядов показывает возможность увеличения концентрации носителей заряда с целью создания атмосферных плазменных локальных образований. Естественная концентрация носителей зарядов в ионосферных слоях $\mathrm{D}, \mathrm{E}, \mathrm{F}$ лежит в пределах от $10^{-2}$ до $10^{-5} \mathrm{~cm}^{3}$. Их распределение по высоте приведено на рисунке 5 .

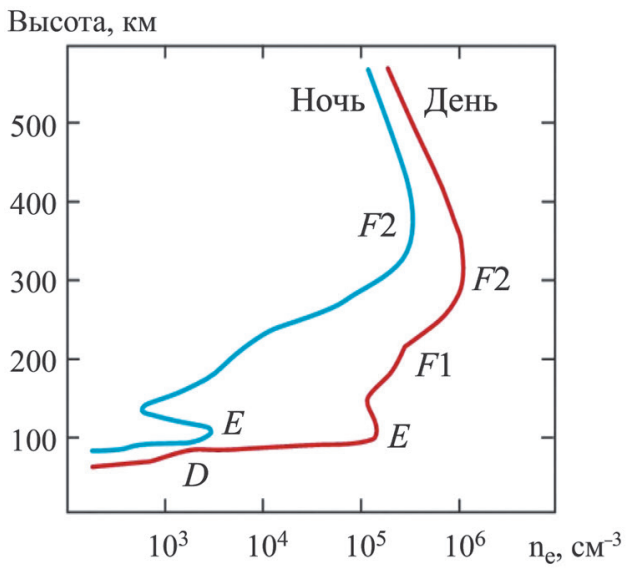

Рис. 5. Распределение концентрации носителей зарядов в ионосферных слоях атмосферы

Таким образом, максимальная концентрация зарядов в атмосфере не превышает $10^{-5}-10^{-6}$. Указанная концентрация достаточна для работы тропосферных радиостанций СВЧ диапазонов, что в частности показал положительный опыт их применения в Чехословакии в 1968 году. Для реализации тропосферных связей в оптических диапазонах требуется значительно большая концентрация носителей зарядов. Создание искусственных плазменных образований на водных аэрозолях может способствовать достижению этой цели. Поэтому для оценки экранирующих свойств заря- женных микрочастиц, возникает необходимость исследования рефлекционных параметров электромагнитного излучения в различных спектральных диапазонах.

В отличии от нормальных условий, имеющих место на земной поверхности, вода в высоких слоях атмосферы обладает свойствами оставаться в жидком состоянии при пониженных температурах. Капли водного аэрозоля могут оставаться в жидком состоянии до температур минус $50-70{ }^{\circ} \mathrm{C}[6]$. Поэтому представляет интерес изучение не только электрофизических, но и термодинамических свойств дисперсных частиц в интервале температур. Такой подход позволяет оценить роль внутримолекулярных и межмолекулярных связей на параметры воды и другие химические соединения [7] в однородном и неоднородном состояниях.

\section{Лuтература}

1. Abrosimov N.I., Bashkatov I.P. International aerosol symposium. Aerosol technology Ltd. Investigation of changed aerosol clustering in the transit space, 1994. $94 \mathrm{c}$.

2. Физические величины: Справочник / А.П. Бабичев и др.; Под. ред. И. С. Григорьева, Е. 3. Мейлихова. М.; Энергоатомиздат, 1991. - $1232 \mathrm{c}$.

3. Паули В. Физические очерки. - М.: Наука, 1975. $256 \mathrm{c}$.

4. Лившии М. Н. Аэроионификация: Практическое применение. М.; Стройиздат, 1990. 168 с.

5. Шпольский Э. В. Атомная физика. - М.: Наука, 1984. $552 \mathrm{c}$.

6. Кондратьев К.Я., Москаленко Н.И., Поздняков Д.В. Атмосферный аэрозоль. Л.: Гидрометеоиздат, 1983.

7. Буш А.А. Физическая химия материалов и процессов электронной техники. Часть І. Электронная структура и свойства химических элементов. Учебное пособие / Моск. гос. техн. университет радиотехники, электроники и автоматики. М., 2014. 136 с. 